\title{
Influence of the Particles Creation on the Flat and Negative Curved FLRW Universes
}

\author{
Giovanni Montani \\ ICRA - International Center for Relativistic Astrophysics \\ INFN-Istituto Nazionale di Fisica Nucleare \\ Dipartimento di Fisica (G9), Università di Roma, "La Sapienza", 00185 Rome, \\ Italy
}

PACS 04.20.Jb, 98.80.Dr

\begin{abstract}
We present a dynamical analysis of the (classical) spatially flat and negative curved Friedmann-Lameître-Robertson-Walker (FLRW) universes evolving, (by assumption) close to the thermodynamic equilibrium, in presence of a particles creation process, described by means of a realiable phenomenological approach, based on the application to the comoving volume (i. e. spatial volume of unit comoving coordinates) of the theory for open thermodynamic systems.

In particular we show how, since the particles creation phenomenon induces a negative pressure term, then the choice of a well-grounded ansatz for the time variation of the particles number, leads to a deep modification of the very early standard FLRW dynamics.

More precisely for the considered FLRW models, we find (in addition to the limiting case of their standard behaviours) solutions corresponding to an early universe characterized respectively by an "eternal" inflationary-like birth and a spatial curvature dominated singularity. In both these cases the so-called horizon problem finds a natural solution.
\end{abstract}




\section{Introduction}

Though the "Standard Cosmological Model" [1, 2] finds its theoretical grounds on a purely classical and maximally symmetric solution of the Einstein equations, like the Friedmann-Lameitre-Robertson-Walker (FLRW) model, nevertheless it is commonly believed that quantum effects could have played an important dynamical role during the evolution of the early universe.

Among such quantum effects are surely of key relevance those related to processes of particles creation; indeed, due to the strong time variation singled out by the background gravitational field near the cosmological singularity, it is naturally expected that quantum fluctuations of the field itself, as well as the dynamics of any quantum field living on such a nonstationary background, lead to phenomena of matter creation, whose existence can in turn modifys the early universe behaviour.

A detailed microscopic description of matter creation phenomena should be provided by a (fully self-consistent) quantum field theory on a curved space-time [3], or, when the backreaction of the created matter becomes no longer negligible (in the sense of strong quantum fluctuations around any average effect), by a pure quantum gravity theory in presence of "matter" fields.

A macroscopic phenomenological description of processes of matter creation, able to make account of the everage effect induced on the (classical) dynamics of an isotropic expanding universe, was proposed by I. Prigogine et al. (see [4]-[6]) which is based on the application, to the universe comoving volume (i. e. spatial volume of unit comoving coordinates), of the standard theory of open thermodynamic systems having a conserved specific entropy (entropy per particle); such a description was then generalized in [7] by allowing the specific entropy be no longer constant and putting its formulation in a completely covariant form.

If the matter present in the universe is described by the energy-momentum tensor of a perfect fluid, then, in such an approach, the pressure of the fluid, apart from the usual thermostatic component, contains an additional term, directly related to the time variation of the particles number within the comoving volume; furthermore the total entropy within such a comoving volume, of the universe, directly proportional to the particles number, is clearly no longer constant (like in the Standard Cosmological Model), since it increases during the processes of matter creation (with interesting implications about the explanation of the actual universe entropy).

Of course, the above mentioned macroscopic phenomenological approach is not completely self-consistent since it can not clearly determine the correct expression for the rate of particles creation; therefore such an information is an open degree of freedom of the scheme and it should be provided by the formulation of an appropriate ansatz for the considered dynamical model.

In [8] the influence of a particles creation term on a FLRW universe, having a flat three-dimensional space, was studied in presence of bulk viscosity, by showing 
how the assumption of a reasonable ansatz (for the rate of particles creation) leads to the appearance of nonsingular cosmologies in correspondence to some particular choice of the free parameters of the model.

Our analysis generalize the ansatz presented in [8] to make it even suitable for the description of a spatially negative curved FLRW model and the resulting early dynamics (analysed close to the thermodynamic equilibrium and in absence of bulk viscosity, which, in agreement to what below discussed in section 2 should be appropriately treated in this framework only as a small perturbative term) appears to be, in both the cases, as deeply modified by the processes of matter creation. Indeed in the case of a flat FLRW universe we get a nonsingular cosmology in correspondence to an open set of the allowed parameters.

More precisely, by choosing an appropriate time variable (the logaritm of the comoving volume), we reduce the dynamical problem to an evolutive equation for the only energy density filling the universe, whose analytical solution provides a functional form of the time variable which is bounded or unbounded depending on the sign of the integration constant. In the physical case of a strictly positive integration constant, in correspondence to any choice of the allowed parameters, the evolution of the universe asymptotically approaches for sufficiently late times the FLRW one, while its early stages are characterized, respectively in the flat and negative curved cases, by an "eternal" inflationary-like behaviour and a spatial curvature dominated singularity.

The most important aim of this paper is to show how, at least in the adopted phenomenological framework, the matter creation could have been deeply influenced the very early phases of the universe life, up to the point of providing an explanation for some fundamental puzzles of the modern cosmology, like the cosmological singularity and the horizon problem.

It is important to observe how, though the precise nature of the phenomena of particles creation relays on the detailed microphysics working in the early universe, nevertheless the notion of heat Big-Bang (indeed we will get below the universe has a maximal initial temperature to be reasonably fitted by a value $\geq 10^{19} \mathrm{GeV}$ ) implies that the most part of the created particles should be initially photons.

In section 2 is given a brief review of the theory of open thermodynamic systems, whose application to the universe evolution provides the theoretical framework for the dynamical analysis developed in section 3; finally concluding remarks follows in section 4 .

\section{Theory of the Open Thermodynamic Systems}

Here we provide a description of the theory of open thermodynamic systems and its application to the universe thermodynamics, which leads to the natural phenomenological description of matter creation processes adopted in the analysis 
of section $3[$ [4]-[6] (see also [7, 8]).

Let us consider a thermodynamical fluid whose energy density, thermostatic pressure, volume, temperature, entropy and particles number are denoted respectively by $\rho, p, V, T, S$ and $N$. All these thermodynamical parameters should satisfy the Gibbs relation:

$$
d(\rho V)=T d S-p d V+\mu d N
$$

where $\mu$ denotes the chemical potential (i. e. the Gibbs function per particle) and is defined as:

$$
\mu=\frac{(\rho+p) V}{N}-\frac{T S}{N}
$$

By the above definition of the chemical potential it is easy to rewrite the relation (11) in the form:

$$
d(\rho V)=N T d \sigma-p d V+\frac{H}{N} d N
$$

where $\sigma$ and $H$ denote respectively the specific entropy $(\sigma=S / N)$ and the total enthalpy $(H=(\rho+p) V)$.

Now, by the first law of thermodynamics, we get the fundamental relation (total energy conservation):

$$
d(\rho V)=\delta Q-P d V
$$

where $\delta Q$ indicates the heat exchanged and $P$ the total thermodynamical pressure.

By the comparison of the two equations (3) and (困) take place the following natural identifications:

$$
\delta Q=N T d \sigma \quad P=p+\Pi
$$

where $\Pi$ denotes an additional pressure term due to the matter creation:

$$
\Pi=-\frac{H}{N} \frac{d N}{d V}=-(\rho+p) \frac{d \ln N}{d \ln V}
$$

When applying this theory to the case of an expanding isotropic universe we should require that all the thermodynamic functions depend only on time and the volume $V$ be the comoving volume corresponding (by convention) to an unit coordinate volume.

Finally, by requiring that the universe be an adiabatic system, we get the following fundamental relation:

$$
\delta Q=0 \Rightarrow N T d \sigma=0
$$


Now, if we treate the universe as a closed system having a fixed number of particles per comoving volume $(N=$ const.), then the above adiabatic condition (7) leads to the entropy conservation $d S=0$ (like in the Standard Cosmological Model); instead when the universe behaves as an open thermodynamic system and it is allowed the particles number per comoving volume changes during its evolution, the same adiabatic relation provides the following fundamental expression for the entropy variation:

$$
T d S=\frac{T S}{N} d N
$$

To put the above thermodynamical scheme in a covariant form (appropriate to our purposes), it is enough to redefine the energy-momentum tensor of a perfect fluid as:

$$
T_{i k}=(\rho+P) u_{i} u_{k}+P g_{i k} \quad i, k=0,1,2,3
$$

where $u_{i}$ and $g_{i k}$ denote respectively the four-velocity of the fluid and the space-time metric.

To conclude, it is important to abserve that, since for $d \ln N / d \ln V>(\rho+$ $3 p) /(3 \rho+3 p)$ we have $\rho+3 P<0$, then, in agreement to [11], the particles creation term can provide a sufficiently negative pressure to remove the cosmological singularity.

Let us now face the question concerning the possibility of including in the above scheme viscosity effects.

Indeed it is commonly believed that phenomena of matter creation should be associated with the presence of a certain degree of bulk viscosity, as assumed in [8]; however, as discussed in [9], when referred to rapidly space-time varying phenomena (like the ones concerning the very early cosmology) the standard theory of viscosity should be amended for accounting of the finite velocity by which any thermodynamical effect propagates.

Such an aim can be reached, in a phenomenological approach, by introducing suitable relaxation times (typically of the order of the mean collision time) which make account for the characteristic time of the system evolution. When such characteristic time is sufficiently large, then the standard theory of viscous fluids can provide a satisfactory description of the considered thermodynamic system; but if such time becomes short enough, the system resembles more a viscoelastic solid and the causal effects should be taken into account.

More precisely, when referred to the only presence of bulk viscosity, the theory of viscoelastic matter, derived in [9], can be (dynamically) summarized by the following energy-momentum tensor:

$$
T_{i k}=(\rho+P+\omega) u_{i} u_{k}+(P+\omega) g_{i k} \quad i, k=0,1,2,3
$$

where $\omega$ denotes the dilatational-stress coefficient and satisfys the equation: 


$$
\omega+\tau_{0} \dot{\omega}=\xi u_{; i}^{i} \quad(\quad) \equiv(\quad)_{; i} u^{i}
$$

with ; indicating the covariant derivative and $\tau_{0}$ and $\xi$ respectively the relaxation time and the dilatation coefficent; in the limit $\tau_{0} \rightarrow \infty$ the ratio $\xi / \tau_{0}$ goes over the bulk modulus.

Since such a theory is only appropriate to describe quasi-stationary systems, which evolve close to the thermodynamical equilibrium, then $\omega$ should fulfills the inequality [10]:

$$
\omega^{2} \frac{\tau_{0}}{\xi} \ll \rho
$$

Now the first law of the thermodynamics (which is provided by the conservation law for the energy-momentum tensor (10) and in the below analysis plays a key dynamical role) reads:

$$
d \rho=-(\rho+P+\omega) d \ln V
$$

Since $\omega, \tau_{0}$ and $\xi$ are expected to be function of the only energy density $\rho$ (below, by choosing a suitable ansatz for the particles creation term, we get that even $P$ is a function of $\rho$ ), then a good (dimensional) assumption about the form the ratio $\xi / \tau_{0}$ should take, is given by $\mathrm{t}$ :

$$
\frac{\xi}{\tau_{0}}=\rho\left(\frac{\rho}{\rho^{*}}\right)^{k} \quad \rho^{*}=\text { const. }>0 \quad k=\text { const. } \geq 0
$$

Hence equation (12) rewrites:

$$
\omega \ll \rho\left(\frac{\rho}{\rho^{*}}\right)^{\frac{k}{2}}
$$

By the dynamical analysis developed in the next section we will show that, in the case of physical interest, during the all universe evolution, the energy density satisfys the restriction $\rho \leq \bar{\rho} \quad \bar{\rho}=$ const. $>0$.

Now in the cases $\bar{\rho} \ll \rho^{*}$ and $\bar{\rho} \sim \rho^{*}$, by (15), we see that in equation (13) the contribution due to $\omega$ is to be regarded as a small perturbation. Instead in the case $\bar{\rho} \gg \rho^{*}$, the above claim is no longer always true. However the former cases appear preferrable from a physical point of view, since, in the limit of very small values of the viscosity coefficient $\xi$, we can yet speak of a real (weakly) viscous fluid (i. e. are yet allowed large values of the relaxation time $\tau_{0}$ ). But the most important physical reason which leads us to believe the viscosity effects be negligible in the earliest phases of the universe evolution relays on the observation presented at the end of section 1 about the requirement of an early dominant

\footnotetext{
${ }^{1}$ In [10] it was assumed with satisfactory causal results the form $\xi / \tau_{0} \sim \rho$.
} 
radiation component; indeed termalized photons are not expected to resemble a viscous fluid, like instead could take place for a later hadronic component.

According to the above general statement, in our approach we will neglect the viscosity effects which, providing only a small dynamical perturbation, can not drastically modify the results below obtained 4 .

\section{Dynamical Model}

As well known, in a synchronous reference, the geometrical structure of the FLRW universe is summarized by a line element of the form [1]]:

$$
d s^{2}=-c^{2} d t^{2}+R^{2}(t) d l^{2}
$$

where $c$ denotes the speed of the light, $R(t)\left(t_{i} \leq t \leq \infty \quad t_{i} \geq-\infty\right)$ the cosmic scale factor (which has the dimension of a lenght) and $d l^{2}$ the line element of a constant curvature three-dimensional space, i.e.:

$$
d l^{2}=\frac{d r^{2}}{1-k r^{2}}+r^{2}\left(d \theta^{2}+\sin ^{2} \theta d \phi^{2}\right)
$$

Here by $k$ we indicate the curvature signature $(k=-1,0,+1)$.

The evolution of the FLRW universe is governed by the following two Friedmann and "continuity" equations:

$$
\begin{gathered}
H(t)^{2} \equiv\left(\frac{1}{R} \frac{d R}{d t}\right)^{2}=\frac{c^{2} \chi}{3} \rho-\frac{c^{2} k}{R^{2}} \\
d\left(\rho R^{3}\right)=-P d\left(R^{3}\right)
\end{gathered}
$$

Above $\chi=8 \pi G / c^{4}$ (being $G$ the Newton constant).

Now we introduce the new time variable $\eta(t)$, defined by:

$$
d \eta=3 H(t) d t \Rightarrow R(\eta)=R_{0} e^{\frac{1}{3}\left(\eta-\eta_{0}\right)} \quad-\infty<\eta<\infty
$$

where $R_{0}$ and $\eta_{0}$ denote two assigned constants $\left(R_{0}>0\right)$. By other words we adopt as time variable, the logaritm of the comoving volume to describe the universe expansion (the above position $d \eta=3 H(t) d t$ clearly provides an appropriate time variable only in the case of an expanding universe, i. e. $H>0$ ).

In terms of (20) the line element (16) reads:

$$
d s^{2}=-\frac{c^{2} d \eta^{2}}{9 H^{2}(\eta)}+R_{0}^{2} e^{\frac{2}{3}\left(\eta-\eta_{0}\right)} d l^{2}
$$

\footnotetext{
${ }^{2}$ However, on this level, we can not exclude at all that, under certain conditions, the viscosity effects could play a relevant dynamical role during the very early stages of the universe evolution.
} 
Starting by the above line element and in agreement with the thermodynamical scheme presented in the previous section, the dynamical equations for the FLRW model, in presence of particles creation, take the form [1], ]:

$$
\begin{gathered}
H^{2}(\eta)=\frac{c^{2} \chi}{3} \rho(\eta)-\frac{c^{2} k}{R_{0}^{2}} e^{-\frac{2}{3}\left(\eta-\eta_{0}\right)} \\
\frac{d \rho}{d \eta}=-(\rho+P)
\end{gathered}
$$

where $\rho$ denotes the energy density associated with the matter filling the universe, and $P$ the corresponding total (isotropic) pressure, constituted by the following two contributions:

$$
P=p+\Pi \quad \Pi \equiv-(\rho+p) \frac{d \ln N}{d \ln \left(R^{3}\right)}=-(\rho+p) \frac{d \ln N}{d \eta}
$$

Now, to the existence of such dissipative processes is associated an entropy production determined, in agreement with (8), by the relation:

$$
T \frac{d S}{d \eta}=\frac{T S}{N} \frac{d N}{d \eta}
$$

Since, according to the second law of the thermodynamics, the entropy never decreases $(d S \geq 0)$, from (25) we immediately get the conditions:

$$
d N \geq 0 \Rightarrow d \ln N \geq 0
$$

In terms of (24) equation (23) can be rewritten as follows:

$$
\frac{d \rho}{d \eta}=(\rho+p)\left(\frac{d \ln N}{d \eta}-1\right)
$$

By introducing the particles density $n=\frac{N}{R_{0}{ }^{3}} e^{-\left(\eta-\eta_{0}\right)}$ the above equation takes the more compact form:

$$
\frac{d \rho}{d \eta}=\frac{\rho+p}{n} \frac{d n}{d \eta}
$$

The above thermodynamical scheme is completed by the following evolutive equation concerning the universe temperature [7]:

$$
\frac{1}{T} \frac{d T}{d \eta}=\left(\frac{\partial p}{\partial \rho}\right)_{n} \frac{1}{n} \frac{d n}{d \eta}
$$

We conclude the formulation of the considered cosmological problem by observing that the relation between the synchronous time and the adopted time variable $\eta$ is given by: 


$$
t(\eta)=t_{0}+\int_{\eta_{0}}^{\eta} \frac{d \eta^{\prime}}{3 H\left(\eta^{\prime}\right)} \quad t_{0} \equiv t\left(\eta_{0}\right)
$$

as well as, in terms of our variables, the physical horizon reads:

$$
d_{H}(\eta)=c e^{\frac{1}{3} \eta} \int_{-\infty}^{\eta} \frac{d \eta^{\prime}}{3 e^{\frac{1}{3} \eta^{\prime}} H\left(\eta^{\prime}\right)}
$$

The advantage in using the adopted time variable $\eta$ consists of the decoupled character acquired by equation (22) (i. e. since now $R(\eta)$ is a known function, then equations (22) amd (27) decouple from each other [9]), so reducing the considered cosmological problem to solve the only evolutive equation (27).

However to solve the equation (27) it is necessary to assign the equation of state relating the pressure $p$ to the energy density $\rho$, but overall to make an appropriate ansatz for the particles creation term $d \ln N / d \eta$.

In what follows we shall assume an equation of state of the form [13]:

$$
p=(\gamma-1) \rho \quad 1 \leq \gamma \leq 2
$$

where $\gamma$ denotes the adiabatic index (in particular $\gamma$ takes the values $4 / 3$ for the ultrarelativistic matter and 1 for a dust).

In order to individualize an appropriate ansatz for $d \ln N / d \eta$, we preliminary observe that, by using (32), then equation (28) immediately gives:

$$
\rho=\rho_{0}\left(\frac{n}{n_{0}}\right)^{\gamma} \Rightarrow n=n_{0}\left(\frac{\rho}{\rho_{0}}\right)^{\frac{1}{\gamma}}
$$

where $\rho_{0}$ and $n_{0}$ denote two positive constants (namely, $\rho_{0}=\rho\left(\eta_{0}\right) . n_{0}=$ $\left.n\left(\eta_{0}\right)\right)$.

It is easy to realize that equation (33) leads to the following implications:

$$
\begin{gathered}
\rho \equiv 0 \Rightarrow n \equiv 0 \Rightarrow \frac{d \ln N}{d \eta}=0 \\
\rho=\text { const. } \neq 0 \Rightarrow n=\text { const. } \neq 0 \Rightarrow \frac{d \ln N}{d \eta}=1
\end{gathered}
$$

Since in the case of a FLRW model with negative curvature $(k=-1)$ the condition $\rho \equiv 0$ is yet compatible with the existence of a real space-time (characterized by $\left.H=\operatorname{cexp}\left\{-\left(\eta-\eta_{0}\right) / 3\right\} / R_{0}\right)$, we are naturally lead to the following ansatz :

$$
\frac{d l n N}{d \eta}=\alpha\left(H^{2}+\frac{c^{2} k}{R_{0}^{2}} e^{-\frac{2}{3}\left(\eta-\eta_{0}\right)}\right)^{\beta}=\left(\frac{\rho}{\bar{\rho}}\right)^{\beta}
$$

where $\alpha$ and $\beta$ denote two positive constants and we set: 


$$
\bar{\rho} \equiv \frac{3}{c^{2} \chi \alpha^{\frac{1}{\beta}}}
$$

This expression for $d \ln N / d \eta$ clearly satisfys (34) and, in view of (35), if $\rho=$ $\rho_{c}=$ const., we should have:

$$
\rho_{c}=\bar{\rho}
$$

By using (36) and (32), equation (27) can be rewritten in the form:

$$
\frac{d \rho}{d \eta}=\gamma \rho\left[\left(\frac{\rho}{\bar{\rho}}\right)^{\beta}-1\right]
$$

As it is easy to verify the solution of the above equation reads:

$$
\rho(\eta)=\frac{\bar{\rho}}{\left(1+a \bar{\rho}^{\beta} \exp \left\{\gamma \beta\left(\eta-\eta_{0}\right)\right\}\right)^{\frac{1}{\beta}}}
$$

or, equivalently, in terms of $R$ as:

$$
\rho(R)=\frac{\bar{\rho}}{\left[1+a \bar{\rho}^{\beta}\left(\frac{R}{R_{0}}\right)^{3 \beta \gamma}\right]^{\frac{1}{\beta}}}
$$

where $a$ denotes an arbitrary integration constant.

By assigning the initial condition $\rho\left(\eta_{0}\right)=\rho_{0}$, we immediately obtain:

$$
\rho_{0}=\frac{\bar{\rho}}{\left(1+a \bar{\rho}^{\beta}\right)^{\frac{1}{\beta}}} \Rightarrow a=\left(\frac{1}{\rho_{0}^{\beta}}-\frac{1}{\bar{\rho}^{\beta}}\right)
$$

From equations (33), (40) and (42) we get the following expressions for the particles density as well as the particles number:

$$
\begin{gathered}
n=n_{0}\left(\frac{1+a \bar{\rho}^{\beta}}{1+a \bar{\rho}^{\beta} \exp \left\{\gamma \beta\left(\eta-\eta_{0}\right)\right\}}\right)^{\frac{1}{\gamma \beta}} \\
N=n_{0}\left(\frac{\left(1+a \bar{\rho}^{\beta}\right) R_{0}^{3 \gamma \beta} \exp \left\{\gamma \beta\left(\eta-\eta_{0}\right)\right\}}{1+a \bar{\rho}^{\beta} \exp \left\{\gamma \beta\left(\eta-\eta_{0}\right)\right\}}\right)^{\frac{1}{\gamma \beta}} \quad N_{0}=n_{0} R_{0}^{3}
\end{gathered}
$$

Finally, by (22), we get the following key expression for the expansion rate of the universe:

$$
H(\eta)=\sqrt{\frac{c^{2} \chi}{3} \frac{\bar{\rho}}{\left(1+a \bar{\rho}^{\beta} \exp \left\{\gamma \beta\left(\eta-\eta_{0}\right)\right\}\right)^{\frac{1}{\beta}}}-\frac{c^{2} k}{R_{0}{ }^{2}} e^{-\frac{2}{3}\left(\eta-\eta_{0}\right)}}
$$

The expression (40) shows how the energy density of the universe be a nondiverging function of $\eta$ in correspondence to the choice $a \geq 0\left(\rho_{0} \leq \bar{\rho}\right)$, while for 
$a<0\left(\rho_{0}>\bar{\rho}\right)$ it diverges when $\eta$ takes the value $\eta_{f}=\eta_{0}+\left[\ln \left(-1 / a \bar{\rho}^{\beta}\right)\right] / \gamma \beta$. However, for any value of the constant $a$, in the limit $\eta \rightarrow-\infty$, we get $\rho \rightarrow \bar{\rho}$.

As preliminary step, it is important to stress that the two cases $a=0$ (BondiGold steady state universe) and $a<0$, by describing solutions in which the energy density respectively remains constant or increases monotonically (diverging for $\eta=\eta_{f}$ ), do not provide a suitable representation of our actual universe and therefore will be excluded from the following analysis.

Then, it is easy to realize that in the limiting case when the processes of matter creation are absent $(\alpha=0$, i.e. $\bar{\rho} \rightarrow \infty)$, we immediately reobtain, by (45), (30) and (41), the standard FLRW dynamics. Furthermore our solution $(a>0)$ goes over the FLRW dynamics in the limit $\eta \rightarrow \infty$, corresponding to the behaviour:

$$
\rho \propto \frac{1}{R^{3 \gamma}}
$$

Indeed the key difference between the Bondi-Gold steady state model and the our one, consists just of such asymptotic FLRW dynamics, which allows to overcome the two fundamental cosmological inconsistencies of a steady universe, concerning the existence of a microwaves background radiation and the unobserved level of particles creation, as required to keep constant the present energy density of the universe.

More precisely, it is easy to realize that, by a suitable finetuning of the free parameters $\bar{\rho}, \beta$ and $a$, it is possible to fit all the fundamental (predictive) features of the Standard Cosmological Model, including the nucleosynthesis process.

Let us now analyse separately the two cases $k=0$ and $k=-1$ corresponding to the choice $a>0$.

\section{i) Flat FLRW model}

In the case of a flat FLRW model $(k=0)$ the function $H(\eta)$ behaves as the square root of $\rho(\eta)$ and, as it is easy to realize, it vanishes for $\eta \rightarrow \infty$ while for

$\eta \rightarrow-\infty$ approaches the value $c \sqrt{\chi \bar{\rho} / 3}$. Thus, in the limit $\eta \rightarrow-\infty$, we get the following expressions:

$$
\begin{gathered}
\eta-\eta_{0} \sim c \sqrt{3 \chi \bar{\rho}}\left(t-t_{0}\right) \Rightarrow R(t) \sim R_{0} \exp \left\{c \sqrt{\frac{\chi \bar{\rho}}{3}}\left(t-t_{0}\right)\right\} \sim 0 \\
\rho \sim \bar{\rho} \Rightarrow n \sim \bar{n} \equiv n_{0}\left(\frac{\bar{\rho}}{\rho_{0}}\right)^{\frac{1}{\gamma}} \quad N \sim 0
\end{gathered}
$$

In addition it comes out easily from (30) and (31) that the age of the universe and the physical horizon have a diverging character.

Thus we see how the universe emerges from $\eta=-\infty$ by an inflationary-like behaviour characterized by a (nearly) constant value of the energy and particles number densities, to be interpreted as the following asymptotic limits: 


$$
\bar{\rho}=\lim _{\eta \rightarrow-\infty} \frac{U}{V} \quad \bar{n}=\lim _{\eta \rightarrow-\infty} \frac{N}{V}
$$

being $V$ the considered comoving volume $\left(V=R^{3}\right)$ and $U$ the total internal energy in it contained $\left(U \equiv \rho R^{3}\right)$.

By other words, though $U$ and $N$ vanish as $t \rightarrow-\infty$, nevertheless their ratio to the vanishing comoving volume $R^{3}$ approaches constant values.

ii) Negative curved FLRW model

In the case of a negative curved FLRW model $(k=-1)$ we see from (45) how the function $H(\eta)$ vanishes for $\eta \rightarrow \infty$ and diverges for $\eta \rightarrow-\infty$. As it is easy to verify, for $\eta \rightarrow-\infty$ take place the following relations:

$$
\begin{gathered}
\eta-\eta_{0} \sim 3 \ln \left(\frac{R_{0}+c\left(t-t_{0}\right)}{R_{0}}\right) \Rightarrow R(t) \sim R_{0}+c\left(t-t_{0}\right) \sim 0 \\
\rho \sim \bar{\rho} \Rightarrow n \sim \bar{n} \equiv n_{0}\left(\frac{\bar{\rho}}{\rho_{0}}\right)^{\frac{1}{\gamma}} \quad N \sim 0
\end{gathered}
$$

Above, the values $\bar{\rho}$ and $\bar{n}$ should be interpreted in terms of the limits (49).

By (31) it is again easy to realize that the physical horizon diverges even in the case of a negative curved FLRW universe, while by (30) the age of the universe is in this case finite $\sim R_{0} / c+t-t_{0}$.

Since, asymptotically, the energy density approaches the constant value $\bar{\rho}$, then we see how the very early dynamics results to be dominated by the spatial curvature (i. e. $H \propto 1 / R)$.

In both the above cases $(k=0$ and $k=-1)$, since, for $\eta \rightarrow \infty(t \rightarrow \infty)$ the energy density vanishes and the appropriate equation of state corresponds to $\gamma=1$, then. in this limit, we get $N \sim n_{0} R_{0}{ }^{3} a^{-1 / \beta} / \rho_{0}=N_{0} a^{-1 / \beta} / \rho_{0}$.

At the end we observe, how by (25), (29) and (32), the entropy and the temperature of the universe can be expressed respectively in terms of the particles number and number density as follows:

$$
S=S_{0} \frac{N}{N_{0}} \quad T=T_{0}\left(\frac{n}{n_{0}}\right)^{\gamma-1}
$$

By the above expressions for $N(\eta)$ and $n(\eta)$, it is easy to construct the explicit functional form for $S(\eta)$ and $T(\eta)$. In particular it is worth noting how in the two cases $k=0$ and $k=-1$ the entropy reaches for $t \rightarrow \infty$ the (maximum) value $S \sim S_{0} a^{-1 / \beta} / \rho_{0}$, while the (maximum) initial value taken by the temperature is given by:

$$
T_{\text {max }}=T_{0}\left(\frac{\bar{\rho}}{\rho_{0}}\right)^{1-\frac{1}{\gamma}}
$$


In particular for an initial radiation dominated universe $(\gamma=4 / 3)$ we get the maximum value $T_{\max }^{r}=T_{0}\left(\bar{\rho} / \rho_{0}\right)^{1 / 4}$.

Of course the values of the above maximal temperature can be suitably fixed to avoid any contraddiction with the observed universe.

\section{Concluding Remarks}

Finally we enphasize how the analysis of a positive curved FLRW universe $(k=1)$ can not be developed in terms of our approach since the time variable $\eta$ would be hill-defined in the "turning point" of the universe $(H=0)$ (as well as not applicable to the collapsing picture $H<0$ ), naturally expected in the evolution of such a model. Furthermore, as it is easy to realize, for negative values of $H$ the adopted ansatz would lead to a negative synchronous time rate of particles and entropy production, contraddicting the second law of thermodynamics; for these reasons, the analysis of this case requires further investigation.

As the results of our analysis show, the presence of a particles creation term deeply influences the universe dynamics, by allowing the energy density and the temperature have never diverging character. More precisely we get, as above outlined, a drastic departure from the "Standard Cosmology" only in the (very) early stages of the evolution, while for sufficiently later times (depending on the adopted choice of the free parameters), the obtained dynamics approaches the usual FLRW one.

It is worth enphasizing how, as a direct consequences of this departure, the obtained solutions for the two cases $k=0$ and $k=-1$ both provide, by the diverging character of the physical horizon, a solution to the so-called horizon problem.

We conclude by observing how any physical motivation for the adopted ansatz is rather difficult to be provided since it should be referred to an average procedure over all quantum effects being active in the early universe. In particular the impossibility to make precise account for the quantum gravity effect limits strongly the physical derivation of the particles creation rate (indeed, in the framework of our phenomenological approach, the matter is created by the gravitational field itself by its time variation and the main contribution to this average effect can be expected to come from the quantum fluctuations of the cosmological background).

However, in spite of this great difficulties, the relevant influence the matter creation phenomena could have exerted on the very early universe dynamics, we believe, makes of interest any effort in this direction.

Daniele Oriti and one of the two referees are thanked for their valuable advices on this matter. 
This paper was supported, in part by ICRA and mainly by INFN.

\section{References}

[1] E. W. Kolb and M. S. Turner, The Early Universe, (1990), (Adison-Wesley, Reading).

[2] G F R Ellis, Class. and Quantum Grav., (1999), 16, A37.

[3] R N. D. Birrell and P. C. W. Davies, Quantum Fields in Curved Space, (1982), (Cambridge University Press, Cambridge).

[4] I. Prigogine, Thermodynamics of Irreversible Processes, (1961), (Wiley New York).

[5] I. Prigogine et al., Proc. Nat. Acad., (1988).

[6] I. Prigogine et al., Gen. Rel. and Grav., (1989), 21, 767.

[7] M. O. Calvao, J. A. S. Lima and I. Waga, Phys. Lett. A, (1992), 162, 223.

[8] K. Desikan, Gen. Rel. and Grav., (1997), 29 No. 4, 435.

[9] W Israel, Annals of Physics, (1976), 100, 310.

[10] W A Belinskii, E S Nikomarov and I M khalatnikov, Sov. Phys.JETP, (1979), $\mathbf{5 0}(2), 213$.

[11] S W Hawking and R Penrose, 1970 The singularities of gravitational collapse and cosmology, Proc. R. Soc. Lond. A 314, 529.

[12] J Wainwright and G F R Ellis (eds), Dynamical Systems in Cosmology, (1997), Cambridge: Cambridge University Press.

[13] H Friedrich and A D Rendall, The Cauchy problem for the Einstein equations Einstein's Field Equations and their Physical Interpretation, (2000), ed. B G Schmidt (Berlin: Springer) 127, Available as gr-qc/0002074. 